\title{
Belgeo
}

Revue belge de géographie

\section{Le « triangle du lithium » existe-t-il ? Géographie politique d'une régionalisation andine (Argentine, Bolivie, Chili)}

Does the "lithium triangle" exist? Political Geography of an Andean

Regionalization (Argentina, Bolivia, Chile).

\section{Audrey Sérandour}

URL : http://journals.openedition.org/belgeo/43717

DOI : 10.4000/belgeo.43717

ISSN : 2294-9135

Éditeur :

National Committee of Geography of Belgium, Société Royale Belge de Géographie

\section{Référence électronique}

Audrey Sérandour, « Le «triangle du lithium » existe-t-il ? Géographie politique d'une régionalisation andine (Argentine, Bolivie, Chili) », Belgeo [En ligne], 4 | 2020, mis en ligne le 09 novembre 2020, consulté le 11 novembre 2020. URL : http://journals.openedition.org/belgeo/43717 ; DOI : https:// doi.org/10.4000/belgeo.43717

Ce document a été généré automatiquement le 11 novembre 2020.

Belgeo est mis à disposition selon les termes de la licence Creative Commons Attribution 4.0 International. 


\title{
Le « triangle du lithium » existe-t- il ? Géographie politique d'une régionalisation andine (Argentine, Bolivie, Chili)
}

\author{
Does the "lithium triangle" exist? Political Geography of an Andean \\ Regionalization (Argentina, Bolivia, Chile).
}

Audrey Sérandour

1 À l'heure de la mondialisation, la proximité géographique demeure un facteur déterminant de l'intensité des échanges économiques (Hettne, Söderbaum, 2000) et des relations politiques entre pays (Beauguitte, 2010). Les interactions entre sociétés voisines, lorsqu'elles s'intensifient et s'approfondissent, peuvent donner naissance à des espaces régionaux, entendus comme des systèmes fonctionnels regroupant des unités spatiales voisines entre lesquelles les relations, les circulations et les échanges s'intensifient.

2 La formation d'un espace régional peut résulter de différents processus. D'une part, ce peut être le fait d'un régionalisme, c'est-à-dire un acte politique, conscient et planifié (Richard, 2010) de coordination macro-régionale relevant d'une initiative institutionnelle (Nicolas, 2003). Cela est alors formalisé par la signature d'ententes ou d'accords (Mercosur, Unasur, etc.). D'autre part, ce peut être le fait de dynamiques de régionalisation. Celle-ci consiste en une augmentation des flux et échanges entre pays voisins, dans un domaine en particulier (investissements, migrations, infrastructures...), et peut découler aussi bien d'une volonté politique que de pratiques plus spontanées. Depuis les années 1990 et l'intensification des coopérations entre acteurs non-étatiques, un certain nombre d'auteurs se sont intéressés à la prégnance des processus de régionalisation émergeant «par le bas » (Bennafla, 2002; Pasquier, 2003 ; Diouf, 2006). Dans cette perspective du New Regionalism, il ne s'agit pas de nier l'action des gouvernements, ni d'établir une dichotomie rigide entre acteurs, mais bien de prendre en compte les dynamiques bottom-up, davantage spontanées et informelles, 
voire non-intentionnelles, qui ont longtemps été minimisées (Hettne, Söderbaum, 1998). Cet enrichissement des débats sur les dynamiques régionales s'inscrit dans une approche constructiviste des phénomènes sociaux et spatiaux (Väyrynen, 2003).

3 L'Argentine, la Bolivie et le Chili concentrent environ $60 \%$ des réserves mondiales de lithium (BRGM, 2017). Les gisements lithinifères se situent précisément à la triple frontière entre ces trois pays, dans des bassins endoréiques caractérisés par une accumulation de sels et nommés salares. L'ensemble est désigné par l'expression "triangle du lithium». Chacun des angles de ce triangle correspond à un salar en particulier: en Argentine le salar d'Hombre Muerto, en Bolivie le salar d'Uyuni et au Chili le salar d'Atacama.

4 Au-delà de sa vertu descriptive, cette expression a un caractère spatial, car elle suggère une dimension régionale de ces gisements. Elle invite à ne pas penser les salares comme une simple juxtaposition de gisements dispersés entre trois pays voisins, mais comme un ensemble formant un même dispositif spatial transfrontalier et concentrant les plus grandes réserves mondiales de lithium. Pour autant, existe-t-il un espace régional du lithium, à la frontière entre l'Argentine, la Bolivie et le Chili ? La ressource lithinifère et son exploitation sont-elles à l'origine de relations d'interdépendances fortes entre ces espaces voisins donnant lieu à une intégration régionale transfrontalière?

5 Les sociétés produisent l'espace par leurs pratiques, leurs échanges et leurs réseaux, mais aussi par leurs représentations et leurs discours. Aussi, nous prenons le parti de considérer le «triangle du lithium» comme une représentation, c'est-à-dire une construction sociale et mentale d'un schéma de perception et de compréhension du réel. Ce «médiateur de l'expérience spatiale» (Bailly, 1989, p. 54) permet de saisir les relations entretenues par les acteurs avec l'espace et ses ressources. Dès lors, l'usage de l'expression «triangle du lithium» suscite plusieurs questions. Traduit-il le déploiement d'un espace régional transfrontalier du lithium? Quels sont les acteurs pour lesquels cet espace fait sens? Quels acteurs sont à l'origine d'interactions et d'échanges autour du lithium dans cet espace? Les dynamiques que l'on observe dans le «triangle» relèvent-elles d'un régionalisme planifié ou d'une régionalisation spontanée des échanges?

6 L'objectif de cet article est triple. Premièrement, il s'agit de déconstruire la représentation $\mathrm{du}$ " triangle du lithium», afin de saisir les dynamiques spatiales et les logiques d'acteurs qui la sous-tendent. Dans une perspective de géographie politique, nous prêtons une attention particulière aux acteurs, dont les intérêts et stratégies modèlent les représentations et configurations spatiales. En effet, étant pourvus d'intentionnalité, de compétence discursive, de capacité d'action et d'influence sur le monde social, les acteurs agissent sur l'espace en même temps qu'ils sont façonnés par lui. En ce sens, il s'agit d'acteurs spatiaux, dont la relation à l'espace passe aussi bien par des interventions tangibles que par des représentations. Saisir la manière dont se déploie un espace régional implique de prêter attention à ces différents rapports à l'espace. Notons que ces acteurs peuvent être individuels (élus, dirigeants d'entreprises, représentants communautaires...) ou collectifs (États, entreprises, associations, syndicats...), que ces derniers soient informels, formalisés ou institutionnalisés. Deuxièmement, notre réflexion vise à questionner la pertinence de l'échelle macrorégionale dans l'analyse du lithium andin. En effet, les projets lithinifères sudaméricains ont souvent été analysés dans le cadre national (pour l'Argentine, Fornillo, 2015 ; Nacif, Lacabana, 2015 ; pour la Bolivie, Ströbele-Gregor, 2012 ; Olivera Andrade, 
2017 ; et pour le Chili, Gobel, 2013 ; Gundermann, Gobel, 2018). Certains travaux ont questionné les échanges binationaux, mais l'échelle macro-régionale est rarement envisagée. Troisièmement, cet article est une contribution aux réflexions sur la notion de régionalisation, en observant des dynamiques spontanées qui émergent en dehors de tout cadre étatique. En se concentrant sur des pratiques, des réseaux et des échanges non-formalisés, on peut faire l'hypothèse qu'il existe un espace régional du lithium, à la fois infra-national et transfrontalier.

Dans le but de saisir la réalité que recouvre l'expression «triangle du lithium », notre raisonnement sera guidé par le questionnement suivant: au-delà de son caractère descriptif, le syntagme "triangle du lithium » traduit-il ou induit-il une organisation spatiale macro-régionale? Notre hypothèse est que la formule "triangle du lithium » constitue une représentation devenue un enjeu de visibilité pour certains acteurs et un levier d'action pour d'autres, ce qui mène à une forme de régionalisation à la frontière entre l'Argentine, la Bolivie et le Chili.

8 La démonstration s'appuie sur des enquêtes de terrain menées en Argentine, en Bolivie et au Chili entre 2015 et 2018 - temporalité qui exclut du champ de notre analyse les changements politiques survenus en 2019 dans ces trois pays. Afin d'appréhender la manière dont s'est construite la représentation du «triangle du lithium » et de la confronter aux pratiques spatiales dans cet espace transfrontalier, nous avons choisi de mener des entretiens semi-directifs avec une grande diversité d'acteurs. L'objet de notre recherche étant le lithium, en tant que ressource et objet de représentations spatiales, l'identification des acteurs s'est faite par cette entrée: les personnes interrogées sont concernées - de près ou de loin - par le lithium. Pour certains, la relation à cette ressource est évidente : ils en sont les régulateurs (acteurs étatiques, représentés par des institutions nationales ou infra-nationales), il s'agit de leur domaine d'activité (acteurs entrepreneuriaux) ou encore de leur objet de recherche (acteurs universitaires). Pour d'autres, le lien à la ressource est territorial : le lithium constitue une ressource du territoire qu'ils administrent (services déconcentrés, élus locaux...), qu'ils habitent (populations locales, communautés indigènes...) ou qu'ils défendent (acteurs associatifs, ONG, mouvements sociaux...). Cette diversité d'enquêtés nous a permis de saisir un univers de représentations et de significations. Bien entendu, certains acteurs nous ont été plus accessibles que d'autres. Ainsi, l'accessibilité aux populations locales a été contrainte par l'offre de transports dans ces régions de marge, faiblement desservies. En revanche, nous avons pu rencontrer de nombreux représentants étatiques, à tous les échelons, ce qui nous a permis d'approfondir nos questionnements sur les projets de régionalisme du lithium et leur réception dans chaque pays. Le matériau empirique ainsi récolté est composé de plus de 170 entretiens et d'observations de terrain. Nous avons procédé à une analyse thématique du corpus d'entretiens, complétée par un examen de littérature grise, et en particulier celle produite par les entreprises extractives et les États.

9 Nous présenterons les résultats de notre analyse en trois temps. D’abord, en analysant et en déconstruisant la représentation du "triangle du lithium », nous montrerons quels acteurs la portent, dans quel intérêt et à quelle échelle. Puis, nous verrons quels projets de régionalisme ont accompagné cette représentation d'un espace régional. Enfin, nous observerons les dynamiques de régionalisation "par le bas ", qui participent à l'organisation de cet espace. 


\section{Le « triangle du lithium », une représentation mondialisée}

\section{Intérêt mondial pour une ressource concentrée dans un espace frontalier}

Depuis le début du XXI ${ }^{\mathrm{e}}$ siècle, la consommation mondiale de lithium ne cesse d'augmenter : en moyenne $8 \%$ par an entre 2004 et 2014 (Hache et al., 2018, p. 29). Cette demande provient principalement du secteur des batteries, bien que ce métal ait aussi des applications dans l'élaboration de céramiques et de verres, dans la fabrication de graisses, de lubrifiants et d'alliages aéronautiques ainsi que dans l'industrie pharmaceutique. En effet, le lithium dispose d'une importante capacité à stocker les charges électriques et les accumulateurs ion-lithium constituent aujourd'hui l'une des technologies les plus efficientes pour le stockage de l'énergie électrique. Le lithium intervient dans la fabrication de batteries d'appareils électroniques, mais aussi dans le secteur des véhicules électriques et des dispositifs de stockage stationnaire de l'électricité produite par les centrales éoliennes ou photovoltaïques.

11 Dans le contexte de la transition énergétique, qui vise à sortir de la dépendance aux ressources fossiles en se fondant sur le développement de nouvelles technologies de production et de consommation d'énergie (Cacciari et al., 2014), le lithium acquiert un rôle clé (Sérandour, Magrin, 2019). Le stockage de l'énergie constitue une dimension essentielle de cette transition. Ainsi, de nombreux pays cherchent à sécuriser leurs approvisionnements en lithium, comme la Chine, qui développe une stratégie énergétique visant à bâtir une "société durable » (Alexeeva, Roche, 2014), ou l'Union européenne, qui réfléchit aux défis posés par les matières premières nécessaires à cette transition (STRADE, 2018). Dans ce cadre, les gisements lithinifères sud-américains attisent les convoitises, non seulement parce qu'ils concentreraient $60 \%$ des réserves mondiales, mais également parce qu'ils sont qualitativement intéressants (forte teneur en lithium, équilibre physico-chimique facilitant l'exploitation, conditions météorologiques favorables, etc.).

Nos recherches sur le syntagme "triangle du lithium » ont montré que sa première occurrence écrite in extenso date de $2008^{1}$, ce qui est récent et correspond à la période d'intérêt grandissant pour les salares sud-américains. La formule est née dans un cabinet-conseil basé en France ${ }^{2}$, avec comme objectif de désigner ces gisements de manière simple et évocatrice. Dès l'année suivante, cinq publications reprennent l'expression: une étude de la Commission chilienne du cuivre ${ }^{3}$, un article de Courrier International ${ }^{4}$, une note de l'ONG étasunienne $\mathrm{COHA}^{5}$, un article de la revue chilienne Capital $^{6}$ et un mémoire de master soutenu à la Duke University ${ }^{7}$. Puis, à partir de 2010, le syntagme se diffuse rapidement dans différents milieux : la sphère médiatique, aussi bien dans les médias internationaux que nationaux et locaux; les arènes globales, à travers des rapports d'ONG, d'institutions onusiennes et de banques de développement; le monde académique, dans les thèses et mémoires d'étudiants étasuniens, européens et sud-américains ; et la sphère gouvernementale, surtout dans les trois pays concernés.

L'expression «triangle du lithium » constitue une représentation exogène, née hors de l'espace concerné et ensuite largement diffusée, par l'intermédiaire d'une série d'acteurs qui se la sont réappropriée. Les échelles régionale et mondiale se révèlent 
intrinsèquement liées dans l'existence de cette représentation d'un espace régional : ce sont des logiques mondiales de demande en ressources qui produisent une requalification spatiale à l'échelle macro-régionale. Cette requalification centrée sur le gisement donne à celui-ci une existence au-delà des ensembles nationaux qui le composent, supplantant la réalité géologique des salares qui s'organise davantage selon une structure linéaire, le long de la Cordillère des Andes (figure 1).

Figure 1. Le " triangle du lithium » : une représentation géographique triangulaire supplantant la structure linéaire des gisements.

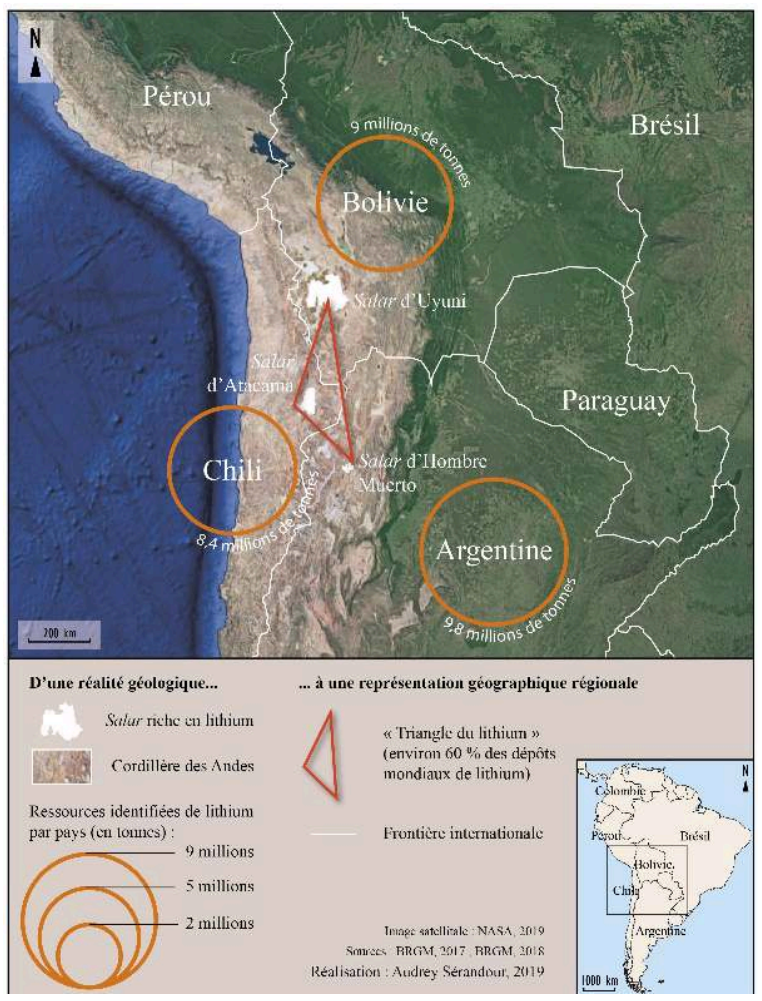

\section{La représentation du « triangle » : enjeu de visibilité}

L'expression «triangle du lithium » impose une certaine représentation de cet espace. Or, aucune représentation n'est neutre; chacune procède d'un processus de construction et d'interprétation, qui exprime l'espace tout en le faisant exister (Saïd, 1980 ; Bailly, 1989 ; Staszak, 2013). Toute représentation véhicule ainsi une subjectivité et nourrit des visions du monde, des intérêts, voire des projets d'acteurs (Lacoste, 1993). Considérer le «triangle du lithium » comme une représentation nécessite donc de s'interroger sur les intérêts liés à son usage, les acteurs qui en bénéficient et les relations de pouvoir associées. En rendant un espace identifiable, l'expression lui confère une visibilité, mais également une identité, qui peut se décliner en image de marque pour certains ou en objet d'attention pour d'autres. Trois principaux types d'acteurs emploient la formule : les acteurs exogènes, les entreprises extractives et les gouvernements sud-américains. Les acteurs exogènes sont à la fois les cabinets-conseil, les médias internationaux et les institutions internationales. Leur utilisation de l'expression «triangle du lithium» vient d'un besoin de schématisation, de simplification dans leur appréhension de cet espace. Nous allons davantage nous 
intéresser aux deux autres catégories d'acteurs, parce qu'ils sont directement présents dans le «triangle».

Sur leurs sites Internet et dans leurs brochures commerciales, les entreprises portant des projets d'exploitation des salares andins usent fréquemment de l'expression «triangle du lithium». Sur les cartes de localisation qu'elles proposent, cette figure triangulaire apparaît souvent comme l'un des seuls repères géographiques à l'échelle de l'Amérique du Sud.

Figure 2. Le " triangle du lithium », un véritable repère géographique. Capture d'écran d'une carte proposée par une entreprise développant un projet d'exploitation de lithium en Argentine (capture effectuée en février 2019).

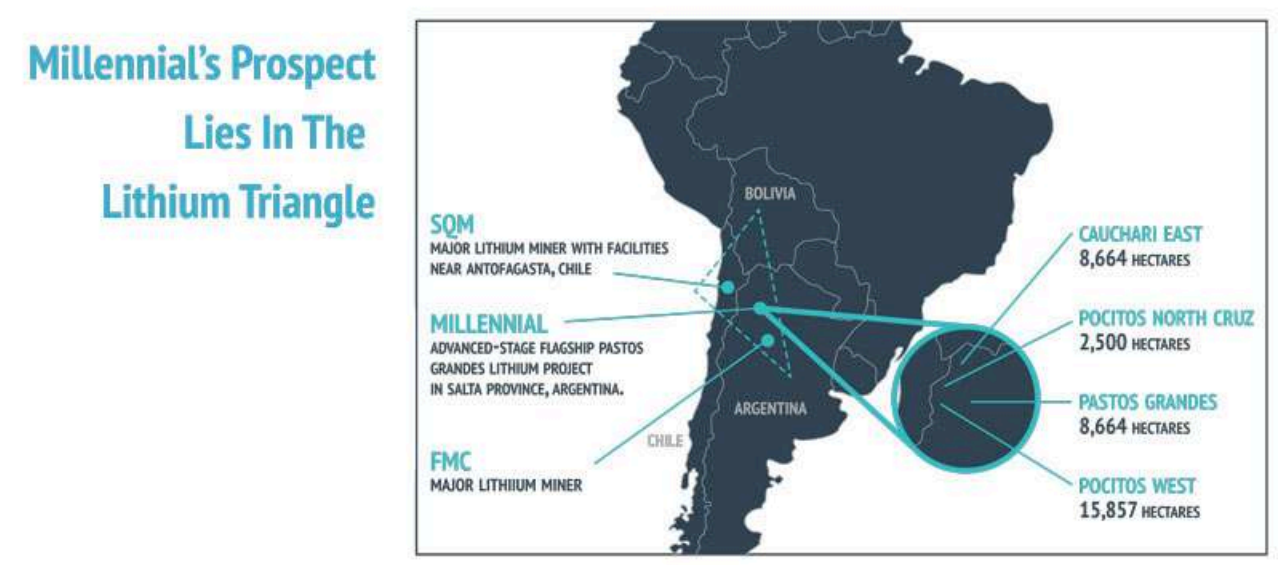

Inscrits dans une logique économique, ces acteurs cherchent à se positionner sur un marché. Leur utilisation de la figure du «triangle » montre que ces entreprises veulent assurer à leurs investisseurs et à leurs clients qu'elles se situent dans l'espace le mieux doté en ressources lithinifères de la planète. S'établir dans le "triangle du lithium » constitue un gage de qualité, car les saumures y sont très concentrées en lithium, et une promesse de longévité, car les gisements sont conséquents et assurent donc une durée de vie confortable aux projets d'exploitation. La représentation agit ainsi comme une image de marque.

Du côté des gouvernements - nationaux, mais aussi infra-nationaux -, on retrouve cet enjeu de visibilité. Pour l'Argentine, la Bolivie et le Chili, faire partie du "triangle » constitue une aubaine: cet espace macro-régional dispose d'une renommée internationale et d'une identité attractive qui attirent les investisseurs. Les représentants étatiques sud-américains emploient généralement l'expression « triangle $\mathrm{du}$ lithium» pour faire référence à l'importance quantitative des gisements. L'inscription dans cet espace constitue un critère d'attractivité économique pour ces pays.

18 Les gouvernements infra-nationaux cherchent également à tirer parti de la popularisation de l'expression "triangle du lithium ». En Argentine - pays fédéral où les ressources naturelles relèvent des provinces - les autorités provinciales identifient le «triangle » à une position stratégique, un référentiel auquel elles cherchent à se faire associer. Ainsi, la découverte d'un nouveau gisement au Sud du salar d'Hombre Muerto a conduit les autorités de la province de Catamarca à affirmer que le «triangle $\mathrm{du}$ lithium» s'était agrandi vers le $\mathrm{Sud}^{7}$, à la manière d'un front pionnier. Cet 
agrandissement a pour effet d'inclure pleinement la province de Catamarca dans le " triangle ", ce qui n'est pas le cas si seul le salar d'Hombre Muerto est considéré.

La représentation du «triangle du lithium » offre une visibilité à l'échelle mondiale aux entités territoriales qui le composent. Comme le formule un ancien directeur provincial des mines de Jujuy : «ça nous permet d'exister sur la carte ${ }^{8}$. Les États sud-américains emploient donc largement l'expression, participant à entretenir cette représentation d'un espace régional. Pour autant, cet usage s'accompagne-t-il de l'émergence d'un régionalisme du lithium? Traduit-il une forme de régionalisation des pratiques et circulations liées à cette ressource?

\section{Les limites du régionalisme}

\section{Quels projets politiques de régionalisme entre l'Argentine, la Bolivie et le Chili?}

L'appropriation de l'expression "triangle du lithium» par les acteurs étatiques argentins, boliviens et chiliens s'est traduite par la formulation de propositions de régionalisme, émanant d'acteurs institutionnels.

21 En 2014, le ministre des mines argentin propose la création d'une Organisation des pays producteurs de lithium ${ }^{9}$ (Opproli). Une telle alliance inter-gouvernementale entre l'Argentine, la Bolivie et le Chili permettrait de représenter et défendre les intérêts de ces pays sur la scène internationale, de peser sur les prix mondiaux du lithium, voire d'unir les efforts pour l'industrialisation de la ressource. Cette proposition stratégique émerge dans le contexte mondial d'accroissement de la demande en lithium, qui alimente des débats sur la manière de maximiser les avantages de l'exploitation de manière coordonnée et concertée au sein du «triangle du lithium » (Castello, Kloster, 2015, p. 72). La volonté politique de structuration d'un ensemble régional répond à un contexte de compétition économique à l'échelle mondiale.

Toutefois, malgré l'amorce d'un dialogue entre les gouvernements, l'Opproli peine à voir le jour. Ce blocage s'explique en partie parce que la Bolivie n'est pas encore entrée en production. Par ailleurs, des difficultés proviennent de contingences politiques: chacun des trois pays dispose de sa vision de l'extraction et de l'attribution des concessions minières (Slipak, 2015) et développe son modèle d'exploitation (Castello, Kloster, 2015). Ces modèles se révèlent difficilement conciliables : alors que l'Argentine incite les transnationales à investir dans le pays, avec par exemple la suppression des taxes sur les exportations minières en 2016, la Bolivie développe au contraire un projet d'exploitation étatique. Enfin, d'un point de vue économique, les trois pays se positionnent davantage comme des concurrents qu'en tant qu'alliés.

Outre le projet Opproli, des réflexions ont émergé au sein d'autres instances politiques régionales telles que le ZICOSUR (Zone d'intégration du centre-ouest de l'Amérique du Sud $)^{10}$. Née en 1997, cette organisation de dimension sous-régionale implique des gouvernements infra-nationaux parmi lesquels se trouvent tous les territoires lithinifères. Elle vise à intégrer ces territoires périphériques aux échanges mondiaux, par le développement du commerce, la formation de ressources humaines et par la construction d'axes de communication (ZICOSUR, 2018). Dans ce cadre, le lithium a été placé à l'agenda du ZICOSUR (Arévalo Moschell, 2014) et des propositions ont été 
formulées autour de la quantification des réserves de la région, de la formation de personnel technique ou encore de la coordination des efforts productifs. Cependant, là encore, les réflexions demeurent à l'état de proposition.

\section{Une nationalisation du débat sur la régulation du lithium}

24 Aborder des processus régionaux en géographe nécessite de considérer avec attention les jeux d'échelles à l'œuvre. Entendues comme des constructions sociales (Brenner, 2001 ; Marston, 2000 ; Masson, 2009), les échelles résultent de pratiques et de projets d'acteurs. La construction d'un espace régional formel autour du lithium nécessite donc que les acteurs gouvernementaux s'investissent dans ce projet d'intégration. Or, les gouvernementaux argentins, boliviens et chiliens développent davantage des projets et visions territoriales à l'échelle nationale, déployant leurs efforts dans la consolidation des cadres de régulation nationaux, voire infra-nationaux pour les provinces argentines.

25 Cette concentration sur l'échelle nationale s'explique de plusieurs manières. D'abord, l'Argentine, la Bolivie et le Chili présentent des visions idéologiques distinctes, comme nous l'avons mentionné précédemment. Le rôle de l'État dans la régulation de la ressource est appréhendé différemment dans chacun des trois pays. Ensuite, et il s'agit là d'un élément clé, les débats politiques sur la régulation du lithium demeurent ouverts dans chacun des pays formant le «triangle du lithium ». Au Chili, par exemple, le gouvernement a créé la Commission nationale du lithium en 2014 ; cette Commission consultative ministérielle a été chargée de dresser un état des lieux et d'élaborer une politique nationale du lithium. À partir du rapport qu'elle a rendu en 2016, le gouvernement a entamé un processus de réflexion sur les modalités de mise en place de cette politique du lithium. Toutefois, ces modalités demeurent en débat dans la société chilienne, divisée entre les tenants du néolibéralisme et les partisans de la nationalisation. Au-delà de ces réflexions sur le cadre de régulation en lui-même, l'intérêt mondial pour le lithium sud-américain et l'intensification de son exploitation suscitent aussi des débats socio-politiques sur les choix de modèle de développement: faut-il approfondir le modèle extractiviste néolibéral ou rechercher davantage de souveraineté sur les ressources minières ? Le débat reste ouvert.

Par ailleurs, en Bolivie le projet d'exploitation du lithium s'inscrit dans une dynamique de construction nationale, qui place les ressources naturelles au cœur du destin du pays (Sérandour, 2017). Le projet national prime sur les perspectives régionales.

Enfin, des différends géopolitiques participent à l'impulsion de dynamiques centrifuges au sein du «triangle du lithium». Préoccupés par l'affirmation de leur souveraineté, ces pays voisins voient leurs relations marquées par une défiance réciproque (Negrete Sepulveda, Velut, 2006; Velut, 2009; Valdès, 2011), renforcée par le souvenir traumatisant de la guerre du Pacifique. Les espaces de marge situés à la triple frontière entre l'Argentine, la Bolivie et le Chili constituent davantage des enjeux de souveraineté que des espaces de rapprochement.

28 Finalement, le repli sur l'échelle nationale révèle une contradiction entre deux logiques territoriales marquantes en Amérique du Sud. D'un côté, la volonté d'intégration continentale, qui marque historiquement cet espace et qui a été remise à l'agenda politique dans sa version post-néolibérale par les gouvernements progressistes dans les années 2000 (Amilhat Szary, 2007 ; Auroi, 2009; Riggirozzi, Tussie, 2012). Et de l'autre 
côté, les logiques nationales, portées par la prégnance de l'État-nation dans la tradition géopolitique du continent (Volvey et al., 2006; Perrier Bruslé, 2014). Ce conflit d'échelles classique ralentit le régionalisme (Palle, 2018). Pour les États, l'échelle de référence pour penser le lithium demeure nationale (voire infra-nationale, pour l'Argentine) et leurs actions politiques ne donnent pour l'instant pas lieu à la formation d'un espace régional. Cependant, l'absence de régionalisme institutionnalisé entre l'Argentine, la Bolivie et le Chili ne remet pas en cause la pertinence de l'échelle régionale dans la géographie politique du lithium andin, car un espace régional peut également naître de dynamiques davantage spontanées et informelles.

\section{Une régionalisation « par le bas »?}

\section{La construction d'un espace régional par des acteurs non-étatiques}

29 Afin de se départir d'une vision stato-centrée de la construction des espaces régionaux, les tenants du New Regionalism invitent à considérer les signes de régionalisation des échanges, des circulations et des pratiques d'acteurs non-étatiques. Prêter attention à ces dynamiques moins formalisées permet d'entrer dans la complexité des formes sociales et spatiales que peut prendre un espace régional. En effet, ce dernier constitue une véritable construction sociale, qui émerge d'un agencement complexe de pratiques, de discours, de relations et de réseaux (Paasi, 2010). Son existence ne relève donc pas forcément d'une construction formelle, elle peut découler de pratiques discrètes, voire banales.

En ce sens, la notion de régionalisation est essentielle pour identifier l'émergence d'espaces régionaux, car elle invite à tenir compte de dynamiques spontanées et informelles, qui ne s'expriment parfois que dans un secteur d'activité. Au sein du " triangle du lithium ", nous avons identifié des acteurs qui opèrent une régionalisation de leurs pratiques ou de leurs réseaux.

Depuis le début de la décennie 2010, en parallèle de l'augmentation de la demande mondiale en lithium et de la multiplication des projets d'extraction dans le «triangle ", la production académique sur le lithium se développe en Argentine, en Bolivie et au Chili. Les équipes de recherche s'élargissent, acquièrent du matériel, approfondissent leurs travaux sur cette ressource. Elles relèvent de divers domaines, allant de la géologie et l'hydrologie à l'écologie politique, en passant par la chimie, l'électrochimie, l'ingénierie des mines et l'ingénierie électrique.

Grâce à la proximité des espaces formant le «triangle du lithium» et malgré les différends géopolitiques prégnants dans la région, les chercheurs des trois pays ont pu se rencontrer et entamer des échanges. Depuis le début des années 2010, des accords bilatéraux ont pris forme entre universités argentines, boliviennes et chiliennes. Mais l'exemple le plus significatif de ces échanges académiques régionaux est celui des séminaires IWLIME (International Workshop on Lithium, Industrial Minerals and Energy). Chaque année depuis 2014, cet événement est organisé conjointement par des universités provinciales d'Argentine, de Bolivie et du Chili et se tient à tour de rôle dans chacun des trois pays (Sérandour, 2018). Il s'agit d'un événement académique international et transdisciplinaire, durant lequel sont présentées des recherches allant de la chimie des matériaux aux technologies de stockage électrique, qui attire aussi quelques représentants d'entreprises extractives. Toutefois, les savoirs y sont 
simplement exposés et découlent rarement de coopérations ou de publications régionales. Si l'intention ayant guidé l'organisation de ces séminaires est de donner vie $\mathrm{au}$ " triangle du lithium » et de créer une dynamique régionale ${ }^{11}$, les IWLIME procèdent pour l'instant d'une régionalisation des réseaux, davantage que des pratiques.

Ces réseaux académiques sont découplés des pratiques des entreprises extractives présentes sur les salares andins, qui disposent de leurs propres dispositifs de recherche, souvent à l'étranger. Pourtant, les entreprises extractives participent également à la création d'un espace régional - principalement entre l'Argentine et le Chili, car la Bolivie ne produit pas encore de lithium. Les axes de circulation, les infrastructures et les réseaux d'acteurs générés par les entreprises incarnent le "triangle du lithium ». La production argentine de lithium est exportée par les ports chiliens, l'entreprise chilienne SQM investit en Argentine, les employés spécialisés dans le secteur lithinifère circulent entre les pays; en somme, un système socio-spatial se met en place au-delà des frontières nationales. Comme dans le domaine universitaire, cette régionalisation des pratiques, des circulations et des réseaux des acteurs entrepreneuriaux donne naissance à une nouvelle structure socio-spatiale organisée autour du lithium.

\section{L'échelle régionale comme levier d'action et d'émancipation?}

À la triple frontière Argentine-Bolivie-Chili, nous avons observé que la multiplication des projets d'exploitation du lithium réactive aussi des réseaux communautaires transfrontaliers. Parmi les différents groupes sociaux vivant dans l'espace qualifié de "triangle du lithium ", certains revendiquent leur appartenance à un peuple préhispanique dépassant les frontières nationales. C'est par exemple le cas des Atacameños, qui organisent régulièrement des rencontres régionales du Pueblo Atacameños sin Fronteras (les Atacameños sans frontières). À l'occasion de l'une de ces rencontres, en 2012, les Atacameños d'Argentine, de Bolivie et du Chili ont ainsi signé la Déclaration de Quetenas, dans laquelle ils s'accordent sur l'unité de leur peuple au-delà des frontières étatiques, ainsi que sur leurs revendications sur les territoires et ressources naturelles (Garcés, Maureira, 2018).

Les projets lithinifères andins réactivent ces revendications, provoquant de nouveaux échanges entre les communautés réparties de part et d'autre des frontières. Ces réseaux permettent aux communautés d'échanger au sujet du lithium, que ce soit pour contester les projets ou pour négocier leur place dans la régulation. Ainsi, depuis 2016, certaines communautés du Nord-Ouest de l'Argentine rencontrent régulièrement des communautés du Nord du Chili, afin de partager leurs expériences de voisinage avec les entreprises lithinifères. Cette régionalisation des réseaux et des échanges autour du lithium s'appuie sur des structures socio-spatiales resignifiées et une identité territoriale renforcée, qui participent à donner corps à l'échelle régionale. Finalement, cette échelle régionale constitue un espace d'action et d'émancipation pour les acteurs communautaires, dont les revendications ont été réactivées dans le contexte politique sud-américain de reconnaissance identitaire et territoriale, qui a débuté dans les années 1990.

L'observation de ces pratiques, échanges et circulations entre l'Argentine, la Bolivie et le Chili, ainsi que la prise en compte des représentations de cet espace transfrontalier rendent l'échelle régionale particulièrement pertinente pour penser la géographie politique du lithium sud-américain. Dès lors, l'analyse des jeux d'acteurs doit intégrer 
cette échelle et prendre en considération les stratégies d'acteurs motivant leur inscription dans des dynamiques régionales.

Pour les acteurs universitaires, les populations locales et les entreprises extractives, la concentration régionale des échanges permet une émancipation des frontières nationales et de logiques telles que la souveraineté sur les ressources ou la concurrence entre Argentine, Bolivie et Chili. De fait, les États nationaux et infra-nationaux ne parviennent pour l'instant pas à s'entendre sur un cadre institutionnel qui permettrait les coopérations académiques; dans ces conditions, les chercheurs se créent leurs propres arènes de dialogue scientifique. Quant aux populations locales, peu audibles sur la scène nationale, elles trouvent à l'échelle régionale un espace d'action à leur portée. Enfin, les intérêts économiques des entreprises extractives les amènent à créer un espace d'infrastructures et de circulations régionales, qui les émancipe des logiques de souveraineté nationale privilégiant les projets nationaux - comme en Bolivie, par exemple.

Pour ces différents acteurs, la régionalisation des échanges constitue la traduction spatiale de processus d'affirmation de leurs intérêts. À d'autres échelles, ils se trouvent en position défavorable dans les rapports de pouvoir, en particulier à l'échelle nationale, où les États occupent un rôle clé.

Les dynamiques de régionalisation observées dans le "triangle " ne répondent pas à une stratégie politique, elles se déploient par des pratiques socio-spatiales, ainsi que des réseaux et circulations d'acteurs. Pour autant, il n'y a pas d'opposition dialectique entre régionalisme et régionalisation ; l'un peut engendrer l'autre, les deux processus se rétro-alimentent. Ainsi, certains universitaires souhaitent institutionnaliser leurs échanges, afin de créer un espace de dialogue entre chercheurs argentins, boliviens et chiliens qui soit pensé sur le long terme et qui puisse constituer un premier pas à l'instauration d'un espace de décision politique régional ${ }^{12}$. Initialement portés par un intérêt scientifique, les chercheurs politisent leur démarche, en impulsant des initiatives devançant les États centraux. Un projet a ainsi été proposé au Fonds d'initiatives communes de l'UNASUR ${ }^{13}$ en 2017, qui vise à développer la recherche sur le lithium, mais également à avancer sur des réflexions politiques, notamment sur les choix de modèles de développement pour ces pays (Sérandour, 2018).

\section{Conclusion}

40 Depuis le début des années 2010, l'augmentation de la demande mondiale en lithium s'est accompagnée de la popularisation de l'expression «triangle du lithium», qui permet de désigner les gisements lithinifères andins. Née hors de l'espace concerné et dans l'articulation avec un contexte mondial, cette représentation d'un espace régional $\mathrm{du}$ lithium a été réappropriée par une diversité d'acteurs au croisement de multiples échelles: régionale, mondiale, nationale, locale. Pour autant, l'existence de cette représentation et son emploi dans les discours d'acteurs ne signifient pas nécessairement qu'elle ait une traduction spatiale. En l'occurrence, durant la décennie 2010, les États argentin, bolivien et chilien ne sont pas parvenus à concrétiser les projets de régionalisme du lithium qui avaient pourtant émergé dans les discussions.

41 Toutefois, l'étude de dynamiques régionales nécessite également de prêter attention à des dynamiques plus discrètes ou informelles. Ainsi, la notion de régionalisation aide à penser la géographie politique du «triangle du lithium », en liant cette représentation 
à des réseaux, des circulations et des pratiques d'acteurs non-étatiques. Cela nous a permis de voir que l'existence de la représentation du "triangle du lithium » a incité des chercheurs à déployer des réseaux transfrontaliers. De même, la proximité des gisements amène les entreprises extractives à construire un système socio-spatial dépassant les frontières nationales. Par ailleurs, des réseaux communautaires se structurent également à l'échelle régionale. Davantage spontanées que le régionalisme, ces dynamiques de régionalisation sont portées par des acteurs qui disposent d'une plus forte capacité d'action sur le court terme que les États; ces derniers étant contraints par la rigidité institutionnelle et une certaine dépendance au sentier (Pierson, 2000).

Au-delà de la représentation, le «triangle du lithium» existe donc au travers des pratiques, échanges, réseaux et discours que la ressource lithinifère permet de construire entre l'Argentine, la Bolivie et le Chili. Pour l'heure, cet espace régional se construit "par le bas", grâce à une proximité géographique, une langue commune d'échanges et le partage d'une représentation de cet espace. Parfois discrètes, ces dynamiques de régionalisation constituent toutefois l'expression tangible d'une intégration régionale qui en est peut-être à ses prémisses.

\section{BIBLIOGRAPHIE}

ALEXEEVA O.V., ROCHE Y. (2014), « La Chine en transition énergétique : Un virage vers les énergies renouvelables ? ", VertigO - La revue électronique en sciences de l'environnement [En ligne], 14, 3, mis en ligne le 28 décembre 2014.

AMILHAT SZARY A.-L. (2007), « Comprendre les référents des projets d'intégration américaine. Du rêve bolivarien aux contre-initiatives anti-globalisation et identitaires contemporaines ", in TAGLIONI F., THÉODAT J.M. (dir.), Coopération et intégration. Perspectives panaméricaines, Paris, L'Harmattan, pp. 209-228.

ARÉVALO MOSCHELLA L.S. (2014), « Integración regional y recursos naturales estratégicos. El caso del triangulo del litio », communication au VII Congreso del IRI / I Congreso del CoFEI / II Congreso de la FLAEI, La Plata (Argentine).

AUROI C. (2009), « Tentatives d'intégration économique et obstacles politiques en Amérique latine dans la seconde moitié du XX $\mathrm{X}^{\mathrm{e}}$ siècle », Relations internationales, 137, 1, pp. 91-113.

BAILLY A. (1989), «L'imaginaire spatial. Plaidoyer pour la géographie des représentations », Espaces Temps, 40-41, pp. 53-58.

BEAUGUITTE L. (2010), « Les votes de l'Assemblée générale de l'ONU de 1985 à nos jours. Pistes (carto)graphiques ", M@ppemonde, 97, http://mappemonde.mgm.fr/num25/articles/ art10104.html.

BECKOUCHE P. (2008), Les régions nord-sud - Euromed face à l'intégration des Amériques et de l'Asie Orientale, Paris, Belin, collection Mappemonde, 220 p. 
BENNAFLA K. (2002), Le commerce frontalier en Afrique centrale. Acteurs, espaces, pratiques, Paris, Karthala, 368 p.

BRENNER N. (2001), « The limits to scale? Methodological reflections on scalar structuration », Progress in Human Geography, 25, 4, pp. 591-614.

BRGM (2017), Fiche de synthèse sur la criticité des matières premières minérales - Le lithium, Paris, BRGM (Bureau de recherches géologiques et minières), 8 p.

CACCIARI J., DODIER R., FOURNIER P., GALLENGA G. \& LAMANTHE A. (2014), « Observer la transition énergétique "par le bas". L'exemple des acteurs du bassin minier de Provence », Métropolitiques [En ligne].

CASTELLO A., KLOSTER M. (2015), Industrialización del litio y agregado de valor local, Buenos Aires, CIECTI, $119 \mathrm{p}$.

DIDELON-LOISEAU C., DE RUFFRAY S. \& LAMBERT N. (2018), « Mental maps of global regions: identifying and characterizing "hard" and "soft" regions ", Journal of Cultural Geography, 35, 2, pp. 210-229.

DIOUF A. (2006), « Afrique : l'intégration régionale face à la mondialisation », Politique étrangère, 4, pp. 785-797.

FORNILLO B. (dir.) (2015), Geopolítica del Litio. Industria, Ciencia y Energía en Argentina, Buenos Aires, Editorial El Colectivo, 213 p.

GAGNON M., FARLEY-CHEVRIER F. (2004), « Chapitre 4. La recherche par Internet », in Guide de la recherche documentaire, Montréal, Presses de l'Université de Montréal, pp. 71-93.

GARCÉS A., MAUREIRA M. (2018), « De familia a organización étnica: redes para una espacialidad transfronteriza en la Puna de Atacama. », Revista Chilena de Antropología, 37, pp. 230-248.

GOBEL B. (2013), « La minería del litio en la Puna de Atacama: interdependencias transregionales y disputas locales », Iberoamericana, XIII, 49, pp. 135-149.

GUNDERMANN H., GOBEL B. (2018), « Comunidades indígenas, empresas del litio y sus relaciones en el salar de atacama », Chungará, 50, 3, pp. 471-486.

HACHE E., SIMOËN M. \& SECK G.S. (2018), Électrification du parc automobile mondial et criticité du lithium à l'horizon 2050, Paris, ADEME, 71 p.

HETTNE B., SÖDERBAUM F. (1998), « The New Regionalism Approach », Politeia, 17, 3, pp. 6-21.

HETTNE B., SÖDERBAUM F. (2000), « Theorizing the Rize of Regionness », New Political Economy, 5, 3, pp. 457-472.

LACOSTE Y. (1993), Dictionnaire de géopolitique, Paris, Flammarion, 1679 p.

MAREÏ N., RICHARD Y. (dir.) (2018), Dictionnaire de la régionalisation du monde, Paris, Atlande, 352 p.

MARSTON S.A. (2000), « The social construction of scale ", Progress in Human Geography, 24, 2, pp. 219-242.

MASSON D. (2009), « Politique(s) des échelles et transnationalisation : perspectives géographiques », Politique et Sociétés, 28, 1, pp. 113-133.

NACIF F., LACABANA M. (2015), ABC del litio sudamericano. Soberanía, ambiente, tecnología e industria, Quilmes, Ediciones del CCC Centro Cultural de la Cooperación Floreal Gorini, 464 p. 
NEGRETE SEPULVEDA J., VELUT S. (2006), « Chili-Argentine : si près, si loin », in LOMBARD J., MESCLIER É. \& VELUT S. (dir.), La mondialisation côté Sud : Acteurs et territoires, Paris, IRD Éditions, pp. 355-372.

NICOLAS F. (2003), « Mondialisation et intégration régionale, des dynamiques complémentaires », Cahiers français, 317 (novembre), pp. 59-63.

OLIVERA ANDRADE M. (2017), La industrialización del litio en Bolivia. Un proyecto estatal y los retos de la gobernanza, el extractivismo histórico y el capital internacional, La Paz, CIDES-UMSA, 332 p.

PAASI A. (2010), « Regions are social constructs, but who or what "constructs" them? Agency in question », Environment and Planning A, 42, pp. 2296-2301.

PALLE A. (2018), «Échelles : à la recherche de l'espace de référence de l'intégration régionale », in MAREÏ N., RICHARD Y. (dir.), Dictionnaire de la régionalisation du monde, Paris, Atlande, pp. 137-139.

PASQUIER R. (2003), « La régionalisation française revisitée : fédéralisme, mouvement régional et élites modernisatrices (1950-1964) », Revue française de science politique, 53, 1, pp. 101-125.

PERRIER BRUSLÉ L. (2014), « L'intégration sud-américaine : des enjeux continentaux aux réalités locales. Un exemple à la tri-frontière Bolivie-Pérou-Brésil », in GANA A., RICHARD Y., La régionalisation du monde. Construction territoriale et articulation global/local, Paris, Édition Karthala et IRMC, pp. 211-231.

PIERSON P. (2000), «Increasing Returns, Path Dependence, and the Study of Politics », The American Political Science Review, 94, 2, pp. 251-267.

RICHARD Y. (2010), L'Union européenne et ses voisins orientaux. Contribution à l'étude des intégrations régionales dans le monde, Mémoire d'HDR (volume 3), Université Paris 1 Panthéon-Sorbonne, 439 p.

RIGGIROZZI P., TUSSIE D. (2012), The Rise of Post-Hegemonic Regionalism. The Case of Latin America, Dordrecht, Springer, $194 \mathrm{p}$.

SAÏD E. (1980), L'Orientalisme. L'Orient créé par l'Occident, Paris, Seuil, 398 p.

SÉRANDOUR A. (2017), « De la ressource naturelle à la construction nationale : analyse géopolitique du projet d'exploitation du lithium du salar d'Uyuni, en Bolivie », Annales de géographie, 1, 713, pp. 56-81.

SÉRANDOUR A. (2018), « Quand les savoirs font ressource : constructions sociales et intégrations territoriales. Une réflexion depuis le "triangle du lithium" (Argentine, Bolivie, Chili) », EchoGéo, 46, [En ligne].

SÉRANDOUR A., MAGRIN G. (2019), « Le lithium andin entre enjeux globaux et attentes locales : chronique d'un malentendu ", in BAGGIONI V., BURGER C., CACCIARI J. \& MANGOLD M., Repenser la transition énergétique. Un défi pour les sciences humaines et sociales, Rennes, Presses universitaires de Rennes, Coll. « Espace et territoires», pp. 191-205.

SLIPAK A. M. (2015), « La extracción del litio en la Argentina y el debate sobre la "riqueza natural" », in FORNILLO B. (dir.), Geopolítica del Litio. Industria, Ciencia y Energía en Argentina, Buenos Aires, Editorial El Colectivo, pp. 91-122.

STASZAK J.-F. (2013), « Représentation de l'espace », in LÉVY J., LUSSAULT M. (dir.), Dictionnaire de la géographie et de l'espace des sociétés, Paris, Éditions Belin, pp. 792-793.

STRADE (2018), Social, economic and environmental challenges in primary lithium and cobalt sourcing for the rapidly increasing electric mobility sector, European Policy Brief, Strategic Dialogue on Sustainable Raw Materials for Europe (STRADE), No. 06/2018, par SCHÜLER D., DOLEGA P., DEGREIF S., 26 p. 
STRÖBELE-GREGOR J. (2012), « Litio en Bolivia. El plan gubernamental de producción e industrialización del litio, escenarios de conflictos sociales y ecológicos, y dimensiones de desigualdad social », desiguALdades.net, Working Paper Series, 14, Berlin, 99 p.

VALDÉS J. G. (2011), « La politique extérieure du Chili : le retour aux origines », Cahiers des Amériques latines, 68, [En ligne].

VÄYRYNEN R. (2003), « Regionalism: Old and New », International Studies Review, 5, pp. 25-51.

VELUT S. (2009), « Argentine-Chili : Une si longue frontière », Confins , 7, [En ligne], mis en ligne le 31 octobre 2009.

VOLVEY A., PERRIER BRUSLÉ L., SOUCHAUD S. \& AMILHAT-SZARY A.-L. (2006), L'Amérique latine, Neuilly-sur-Seine, Atlande, coll. « Clefs Concours, Géographie des territoires », 215 p.

ZICOSUR (2018), ZICOSUR. La zona de integración del Centro Oeste de América del Sur, http:// zicosur.co/acerca-de-zicosur/.

\section{NOTES}

1. Afin d'identifier la première occurrence de l'expression «triangle du lithium » sur Internet, nous avons utilisé les fonctions avancées du moteur de recherche Google. En utilisant l'outil de restriction des résultats par date de publication, nous avons effectué de multiples requêtes sur ce syntagme, en trois langues (français, anglais, espagnol) et sur des périodes de temps affinées en fonction des résultats obtenus. Puis, nous avons consulté et analysé les pages Internet ainsi identifiées. Cette méthode présente des limites : elle se base uniquement sur les pages recensées par Google et ne permet pas d'explorer le Web invisible (Gagnon, Farley-Chevrier, 2004). Nous l'avons donc complétée par une requête directement auprès des acteurs concernés, afin de confirmer nos résultats.

2. Il s'agit de Meridian International Research, dirigé par William Tahil, qui a rédigé un rapport sur le lithium sud-américain en mai 2008, intitulé The Trouble with Lithium 2. Under the Microscope. L'expression «Lithium Triangle » apparaît à trois reprises dans ce document.

3. COCHILCO (2009), Antecedentes para una Política Pública en Minerales Estratégicos: Litio, LAGOS MIRANDA C., octobre 2009.

4. SALAZAR SALVO M. (2009), "Chili. Le lithium dans les mains de la famille Pinochet », Courrier international, $\mathrm{n}^{\circ} 988, \mathrm{p} .18$.

5. COHA (2009), " Bolivia: The myth of the Saudi Arabia of lithium », Council on Hemispheric Affairs, http://www.coha.org/bolivia-the-myth-of-the-saudi-arabia-of-lithium/.

6. Capital (2009), "El litio de la discordia », Revista Capital, 22 décembre 2009, https:// www.capital.cl/el-litio-de-la-discordia-3/.

7. Entretien réalisé avec la géologue du secrétariat des mines, en juin 2017, à San Fernando del Valle de Catamarca.

8. Entretien réalisé avec un géologue et ancien directeur provincial des mines et ressources énergétiques de la province de Jujuy, en novembre 2016, à San Salvador de Jujuy.

9. En espagnol : Organización de Países Prouctores de Litio.

10. En espagnol : Zona de Integración del Centro Oeste de América del Sur. Cette organisation régionale rassemble 47 entités infranationales d'Argentine, de Bolivie, du Brésil, du Chili, du Paraguay et du Pérou.

11. Entretien réalisé avec le directeur de l'un des laboratoires organisateurs du IWLIME, en mars 2018, à Antofagasta. 
12. Entretien réalisé avec le coordinateur du système étatique de science et technologie, en avril 2018, à La Paz.

13. L'Union des nations sud-américaines (UNASUR) est une organisation intergouvernementale dont l'objectif est de développer un espace régional intégré sur le continent sud-américain. L'UNASUR est inspirée du modèle de l'Union européenne. Elle entre toutefois en crise en 2018, à la suite du retrait - temporaire, mais pour une durée indéfinie - de la moitié de ses membres, qui estiment qu'il existe d'importants dysfonctionnements internes.

\section{RÉSUMÉS}

Médias, gouvernements et entreprises extractives emploient de plus en plus l'expression «triangle du lithium » pour désigner les gisements lithinifères sud-américains, répartis entre Argentine, Bolivie et Chili. Au-delà de sa vocation descriptive, cette représentation géographique confère une dimension régionale aux gisements, pourtant rarement interrogée. Afin d'envisager l'existence de l'échelle régionale dans la géographie politique du lithium andin, notre réflexion s'inscrit autour des notions de régionalisme et de régionalisation. L'utilisation de l'expression «triangle du lithium » a-t-elle une traduction spatiale, qu'elle soit engendrée par une volonté politique ou par des pratiques plus spontanées? À partir d'une déconstruction de la représentation mettant au jour les stratégies d'acteurs qu'elle nourrit à différentes échelles, nous analysons les processus de régionalisme inachevés et les dynamiques de régionalisation " par le bas » à l'œuvre dans cet espace transfrontalier.

Media, governments and extractive companies are increasingly using the appellation "lithium triangle" to refer to South American lithium deposits in Argentina, Bolivia and Chile. Beyond its descriptive vocation, this geographical representation gives a regional dimension to the deposits, which is rarely examined. In order to consider the existence of the regional scale in the political geography of the Andean lithium, our reflection is based on the notions of regionalism and regionalization. Does the use of the appellation "lithium triangle" have a spatial translation, whether generated by political will or by more spontaneous practices? Based on a deconstruction of representation that highlights the strategies of the actors at different scales, we analyse the incompleted processes of regionalism and bottom-up dynamics of regionalization existing in this cross-border space.

\section{INDEX}

Mots-clés : Argentine, Bolivie, Chili, géographie politique, lithium, régionalisation, triangle du lithium

Keywords : Argentina, Bolivia, Chili, lithium, lithium triangle, political geography, regionalization 
AUTEUR

AUDREY SÉRANDOUR

Doctorante, Université Paris 1 Panthéon-Sorbonne, UMR 8586 Prodig, Audrey.Serandour@univparis1.fr 